\title{
Differential and Integral Corticosteroid Feedback Effects on ACTH Secretion in Hypoadrenocorticism
}

\author{
H. L. Fehm, K. H. Voigt, G. Kummer, R. LANg, and E. F. Pfeiffer, Department of \\ Internal Medicine, Endocrinology and Metabolism, Center of Internal Medicine \\ and Pediatrics, and Department of Physiology, Center of Biology and Theoretical \\ Medicine, University of Ulm, Ulm, West Germany
}

A B S T R A C T Recent work suggests the existence of a dual corticosteroid feedback mechanism of stressinduced ACTH secretion in the rat. This possibility led us to study the kinetics of suppression of ACTH levels by corticosteroid administration in patients with nonstress ACTH hypersecretion secondary to hypoadrenocorticism. Cortisol was administered according to different protocols, which were chosen to provide extreme variations of the input signal. By this means, two phases of suppression of ACTH levels could be differentiated. A first decrease occurred without latency whenever, and as long as, plasma cortisol levels were rising. There was a linear regression between the logarithm of the increments in cortisol concentrations and the decrease in ACTH levels per minute $(r=0.951)$ (differential or rate-sensitive feedback mechanism). Neither the absolute doses of cortisol, nor plasma cortisol concentrations were closely correlated with the degree of suppression of ACTH by this rapid mechanism. A second decrease in ACTH levels began $\cong 30$ min after corticosteroid administration. In this case there was a significant linear regression between the degree of inhibition of ACTH levels and the cortisol doses $(r=0.997)$ (integral or dose-sensitive feedback mechanism).

The dose-sensitive feedback effects of dexamethasone were less than might have been predicted from its relative anti-inflammatory potency. No rate-sensitive effects were seen with dexamethasone doses of 1.0 or $1.25 \mathrm{mg}$.

\section{INTRODUCTION}

Since the hypothesis of glucocorticoid negative feedback in the hypothalamic-pituitary-adrenal axis was

Portions of the data have been reported at the XIth Acta Endocrinology Congress, Lausanne, Switzerland, 19-23 June 1977, and were published in Acta Endocrinol. Suppl. 212: 259. (Abstr.)

Received for publication 16 November 1977 and in revised form 18 September 1978. proposed in 1947 by Sayers and Sayers (1), many experiments have tested this hypothesis. In 1961, Yates et al. (2) set up the hypothesis that the plasma corticosteroid concentration was regulated by means of a negative feedback controller which performed with a variable set-point. More recently, it could be demonstrated that after the administration of corticosteroids there are two periods of inhibition of stress-induced release of ACTH in the rat (3-9). The first was brief and occurred immediately after the administration of the steroid: this has been designated as "fast feedback." It was characterized by rate sensitivity and saturability. The second period of inhibition of stress-induced ACTH release was much longer and did not occur until an hour or more after steroid administration. The second mechanism required larger doses than the first and the duration of inhibition was prolonged with increased dosage (proportionality). This feedback has, therefore, been called "level-sensitive, delayed feedback." The time in between these two periods was called the "silent period."

These concepts arose mostly from experiments in which the inhibition of the stress-induced ACTH secretion in rats was used to test the state of the hypothalamic-pituitary system. However, nonstress and stress-induced ACTH secretion may be dissociated on the basis of sensitivity to negative corticosteroid feedback effects (10-12). Data from human subjects that bear on the topic of rate-sensitive feedback are scarce. These considerations led us to study the dynamics of suppression of ACTH levels by corticosteroids in patients with nonstress ACTH hypersecretion secondary to hypoadrenocorticism (primary adrenocortical insufficiency). Different experimental designs for administration of cortisol or dexamethasone were chosen to provide extreme variations of the input signal, i.e. magnitude and time-course of cortisol plasma concentrations. The response of the system was evaluated by measuring plasma ACTH concentrations at short time intervals. 


\section{METHODS}

20 patients with primary adrenocortical insufficiency participated in this study. Diagnosis was based on $(a)$ clinical symptoms and signs, $(b)$ demonstration of decreased urinary steroid excretion and plasma cortisol concentrations, $(c)$ failure to respond to ACTH administration on three consecutive days (8-h ACTH stimulation test with 50 IU $\beta^{1-24}$ ACTH [Synacthen, CIBA AG, Wehr, West Germany]), and (d) elevated plasma ACTH levels. Despite adequate replacement therapy, hyperpigmentation to a more or less severe degree persisted in all patients. In most patients, replacement therapy consisted of $37.5 \mathrm{mg}$ cortisone-acetate orally (Cortison CIBA, CIBA AG) (25 $\mathrm{mg}$ in the morning and $12.5 \mathrm{mg}$ in the afternoon) and of 0.1 mg 9, $\alpha$-fluorohydrocortisone (Astonin H, Merck AG, Darmstadt, West Germany) daily in the morning. Patients who were treated with the mineralocorticoid deoxycorticosteronacetate-trimethylacetate (Percorten M, CIBA AG; intramuscular injection of $25 \mathrm{mg}$ every $3 \mathrm{wk}$ ) were excluded from this study for two reasons: $(a)$ deoxycorticosteronacetate-trimethylacetate cannot easily be withdrawn because of its prolonged duration of action and $(b)$ deoxycorticosteronacetate has been shown to antagonize fast-feedback effects of corticosteroids (6). The duration of prior corticosteroid therapy before the study varied from $3 \mathrm{wk}$ to $15 \mathrm{yr}$.

All studies were performed in the morning hours, starting between 8 and 9 a.m., $24 \mathrm{~h}$ after the last dose of the usual replacement therapy. None of the patients experienced any symptoms of corticosteroid withdrawal during this short period. Cortisol (100 mg free alcohol in $20 \mathrm{ml} 50 \%$ ethyl alcohol; Hydrocortison zur Infusion, Hoechst AG, Frankfurt, West Germany) was administered intravenously according to different protocols: Protocol 1 , constant rate infusion of 50 or $12.5 \mathrm{mg}$ cortisol/h for $2 \mathrm{~h}$, with a Braun perfusor; Protocol 2 , infusion of $50 \mathrm{mg}$ cortisol during $15 \mathrm{~min}$ at constant rate; Protocol 3, infusion of $50 \mathrm{mg}$ cortisol during $5 \mathrm{~min}$ at constant rate; Protocol 4, bolus injection of 25 or $5 \mathrm{mg}$ cortisol. It must be stated that $50-100 \mathrm{mg}$ of cortisol is a large intravenous dose when the normal, adult human subject is considered to secrete about $20 \mathrm{mg}$ of cortisol in $24 \mathrm{~h}$. In some cases, dexamethasone-21-orthophosphate (Fortecortin-MonoAmpullen, Merck AG) was given intravenously, either at 1.25 $\mathrm{mg} / \mathrm{h}$ at a constant rate for $2 \mathrm{~h}$, or as a brief infusion of 1 $\mathrm{mg}$ during $15 \mathrm{~min}$. Dexamethasone doses were chosen with respect to their anti-inflammatory potencies; dexamethasone was considered to be 30 times more potent than cortisol (13). Venous blood was drawn from the arm through an indwelling cannula, at 5- or 15-min intervals, for determination of plasma ACTH levels.

Plasma-ACTH was estimated by radioimmunoassay after extraction of ACTH from the plasma as previously described (14, 15). Starting ACTH levels in all patients $24 \mathrm{~h}$ after the last ingestion of corticosteroids were markedly elevated $(1,450.5 \pm 334.3 \mathrm{pg} / \mathrm{ml}$; mean $\pm \mathrm{SEM}$; normal range $0-150 \mathrm{pg} /$ $\mathrm{ml}$ ). Because of the large variations of the starting levels, all values have been calculated as percent of the individual mean base line. Plasma-cortisol measurements were performed by competitive protein-binding assay (16).

Statistical methods (17). To describe the time-course of ACTH levels in response to corticosteroids, two neighboring points of each curve were selected and tested by Student's $t$ test for paired data. As only these segments of the curves were important for generating a hypothesis, it was not attempted to describe the whole curves by statistical analysis. To analyse the relationship between corticosteroids and ACTH levels, a regression analysis was performed for all ACTH values 15 and 75 min after cortisol administration and the corresponding cortisol doses, cortisol concentrations, and changes in cortisol concentrations, respectively.

\section{RESULTS}

The amounts of alcohol necessary in these experiments for dissolution of cortisol were shown to be without effects on ACTH plasma levels. In six patients who received vehicle only, ACTH level varied considerably; however, on the average, there was a continuous slight decrease in normalized levels from $100 \%$, initially, to $80.2 \pm 6.6 \%$ (mean $\pm \mathrm{SEM})$ at the end of the second hour.

When cortisol was infused intravenously at constant rate for $2 \mathrm{~h}$ (Protocol 1 ), there was an immediate and rapid decrease in ACTH levels, which continued throughout the observation period (Fig. 1). The difference between ACTH values at 15 min and the starting values was significant $(P<0.025)$. Plasma cortisol rose rapidly during the first $15 \mathrm{~min}$ of infusion, and then more slowly, reaching a final value of $\cong 60 \mu \mathrm{g} / 100$ $\mathrm{ml}$. With $12.5 \mathrm{mg}$ cortisol/h, a similar time course of ACTH levels was observed, but the degree of inhibition was less pronounced (data not shown).

When $50 \mathrm{mg}$ cortisol were infused during 5 and 15 min (Protocols 2 and 3) a biphasic response of the ACTH levels was observed. An initial, rapid fall in plasma ACTH levels occurred while cortisol concentrations were increasing as a result of the cortisol infusion (Figs. 2 and 3).

Immediately after cessation of the cortisol infusions, ACTH levels rose again, to a maximum 5 min later. In the case of Protocol 3 (50 $\mathrm{mg}$ cortisol in $15 \mathrm{~min}$ ) the

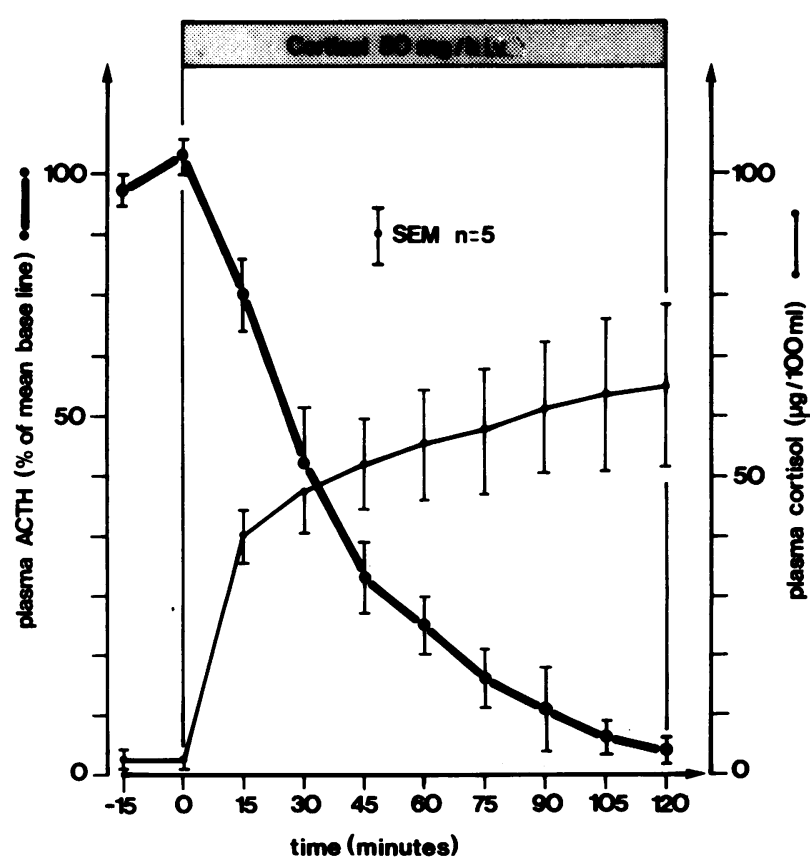

Figure 1 Time course of ACTH levels in response to a constant rate infusion of cortisol in patients with Addison's disease. ACTH values are given as percent of the individual mean starting levels. The difference between ACTH values at 15 min and the starting value was significant $(P<0.025)$. 


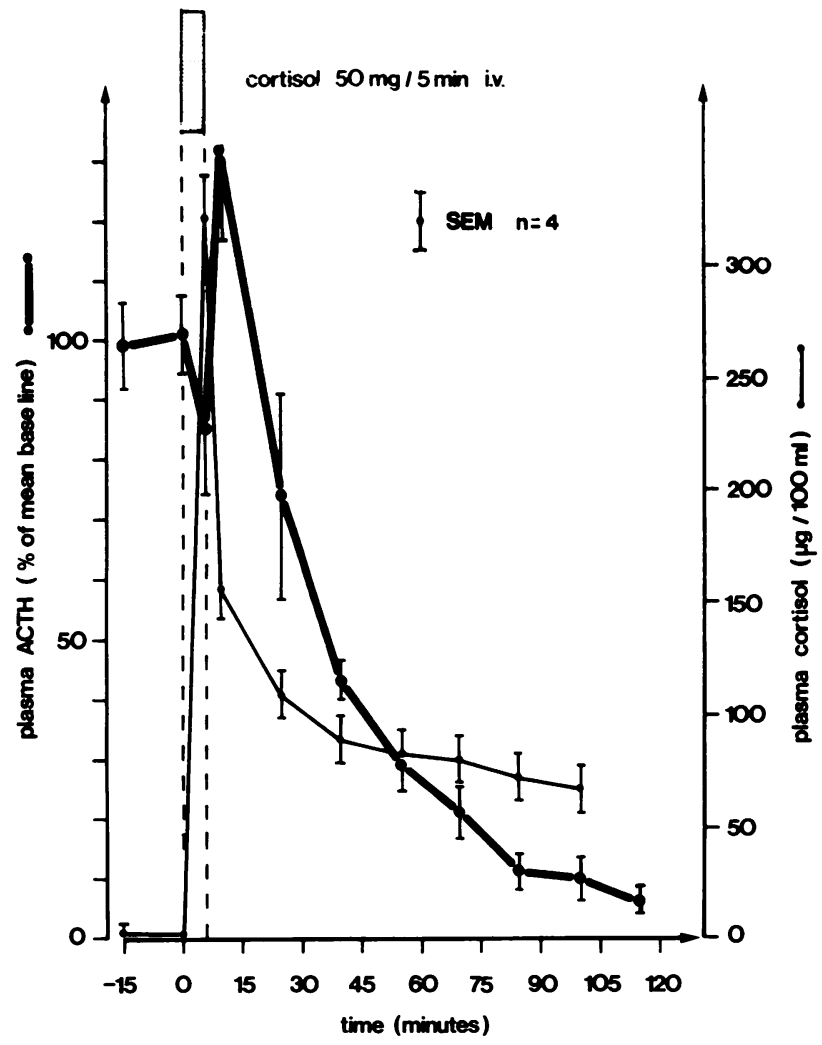

FIGURE 2 Time course of ACTH levels in response to infusion of $50 \mathrm{mg}$ cortisol during $5 \mathrm{~min}$ in patients with Addison's disease.

paradoxical overshoot in ACTH levels from the 15th to the 20th min was significant $(P<0.025)$. Later, a second, continuous decrease in ACTH levels was demonstrable.

The ACTH values $15 \mathrm{~min}$ after injection of $25 \mathrm{mg}$ cortisol as bolus (Protocol 4) were not significantly different from the starting values $(P>0.25)$ (Fig. 4). Plasma cortisol levels were extremely high during this time and still $70 \pm 9 \mu \mathrm{g} / 100 \mathrm{ml}$ (mean $\pm \mathrm{SEM}$ ) at the 15 th min. By 30 min, a continuous decrease in ACTH levels had begun. With $5 \mathrm{mg}$ cortisol given as a bolus, a similar time course of ACTH levels was observed (data not shown). The failure of bolus injections to suppress ACTH levels within 15 min was especially impressive when compared with the significant inhibition observed with Protocol 1 ( $50 \mathrm{mg}$ cortisol/h) after $15 \mathrm{~min}$. In this case, only $12.5 \mathrm{mg}$ cortisol had been infused during this time and cortisol levels had just reached $40 \pm 5 \mu \mathrm{g} / 100 \mathrm{ml}$ (mean \pm SEM) at the end of this period.

From the findings described above, the following hypothesis was generated: After administration of cortisol to patients with Addison's disease, two phases of inhibition of ACTH secretion must be differentiated. A first decline in plasma ACTH levels occurred without delay whenever, and as long as, cortisol levels were rising (rate-sensitive or differential mechanism). A second mechanism with a delay time of $\cong 30$ min may depend upon the cortisol dose (dose-sensitive or integral mechanism). When cortisol was infused at constant rate for $2 \mathrm{~h}$, both mechanisms overlapped temporally. This overlap resulted in a continuous fall in ACTH levels throughout the $2 \mathrm{~h}$. In experiments in which cortisol was infused during short time intervals, the two mechanisms were demonstrated sequentially because of their temporal separation. When cortisol was injected as a bolus, the time of increase in plasma cortisol was too short to activate rate-sensitive feedback elements. Thus only dose-sensitive feedback effects occurred.

To test this hypothesis, a regression analysis was performed, which compared ACTH values 15 and 75 min after the cortisol administration with the corresponding cortisol doses, cortisol concentrations, and changes in cortisol concentrations. The 15 -min values represented the early phase of inhibition, and the 75min values the later phase. There was a significant linear regression between the logarithm of the rate of increase in cortisol concentrations per minute (cortisol

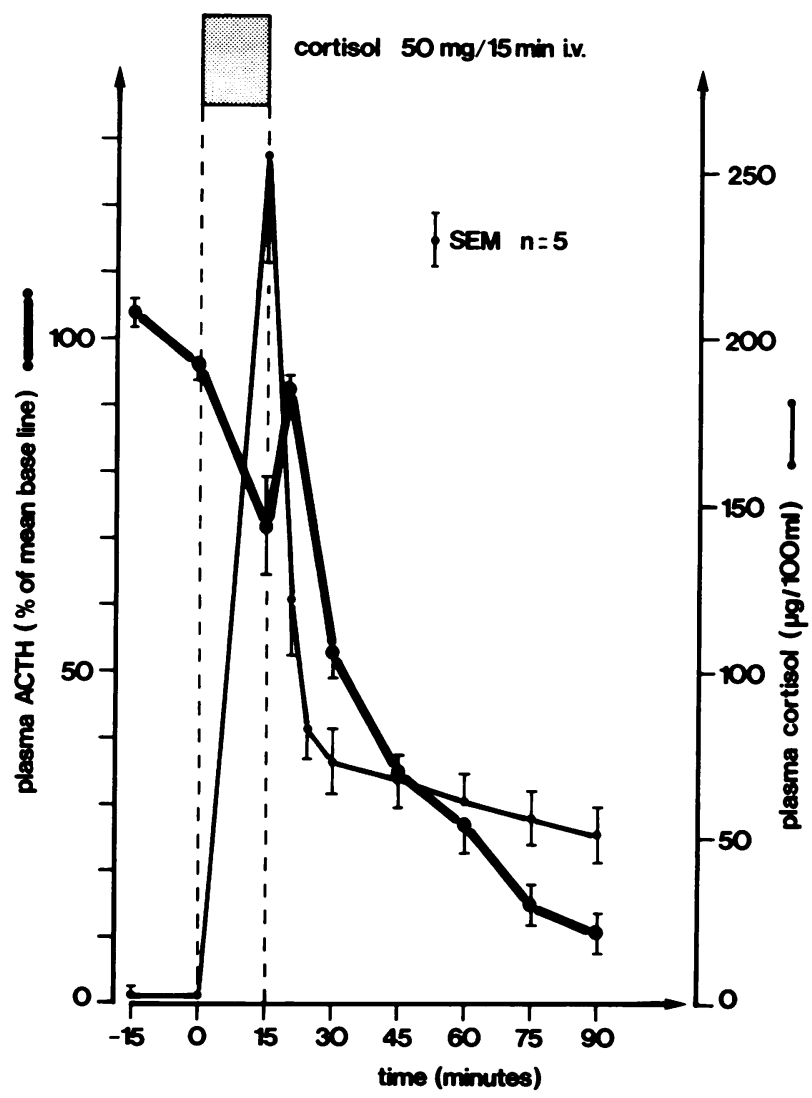

Figure 3 Time course of ACTH levels in response to infusion of $50 \mathrm{mg}$ cortisol during $15 \mathrm{~min}$ in patients with Addison's disease. The difference between ACTH values at 15 and 20 min was significant $(P<0.025)$. 


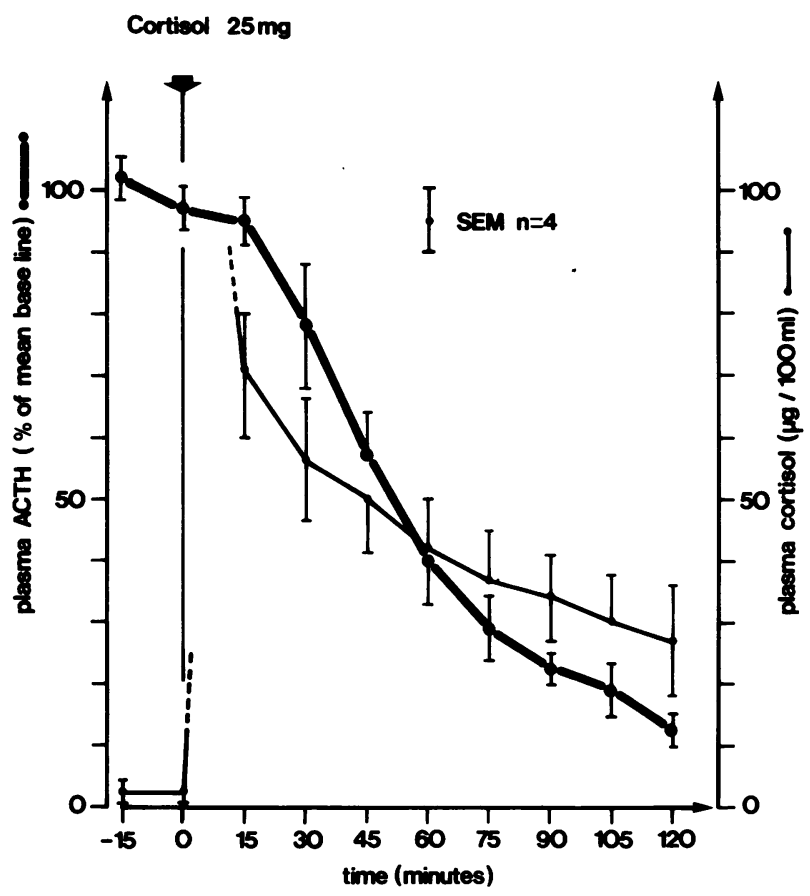

Figure 4 Time course of ACTH levels in response to bolus injection of $\mathbf{2 5} \mathrm{mg}$ cortisol in patients with Addison's disease. Despite extremely elevated cortisol levels, no decrease of ACTH levels occurred during the first $15 \mathrm{~min}$ after administration.

concentration at the end of the infusion period minus starting levels divided by the interval; $\Delta$ cortisol per minute) and the corresponding decrease in ACTH levels per minute (ACTH starting level minus ACTH levels at the end of the infusion period divided by the interval; $\triangle$ ACTH per minute $)(r=0.951,2 \alpha<0.01)$ (Fig. 5). When the cortisol infusion period exceeded $15 \mathrm{~min}$ (as with protocol 1), only the interval between 0 and 15 min was considered. The minimal effective rate of increase in cortisol levels was estimated to be $\cong(0.6 \mu \mathrm{g} / 100 \mathrm{ml}) / \mathrm{min}$ by plotting the data observed with vehicle only on the regression line. According to the above hypothesis, the results with bolus injections were omitted in this correlation. If it were assumed that the ACTH decrease during the first 15 min correlated with $(a)$ the final cortisol levels or with (b) the cortisol dose administered during this time, there would be no reason for omitting the bolus data. However, in these cases the correlations were less strong $([a] r=0.862,2 \alpha>0.05$; and $[b] r=0.832$, $2 \alpha>0.05)$.

The second decrease in ACTH levels, which began $\cong 30$ min after the corticosteroid administration, correlated with the cortisol dose only. There was a linear regression between the decrease in ACTH values 75 min after cortisol administration and the cortisol dose administered during this time, irrespective of the pro- tocol ( $r=0.997 ; 2 \alpha<0.001$ ) (Fig. 6). There was a poor correlation to cortisol concentrations at $75 \mathrm{~min}(r$ $=0.855 ; 2 \alpha>0.05$ ) and obviously no correlation to the rates of changes in cortisol concentration.

In Fig. 7, the effects of dexamethasone ( $1 \mathrm{mg} / 15 \mathrm{~min}$ ) on ACTH levels are demonstrated. There were no definite effects on ACTH levels during the first 15 min. An almost identical time course of ACTH levels was observed with infusion of $1.25 \mathrm{mg}$ dexamethasone $/ \mathrm{h}$ at a constant rate (data not shown). Apparently, these doses of dexamethasone did not suffice to activate ratesensitive feedback elements. When the inhibition observed at $75 \mathrm{~min}$, was compared with that obtained with different doses of cortisol, it appeared that $1 \mathrm{mg}$ dexamethasone caused about the same dose-sensitive inhibition of ACTH levels as did $15 \mathrm{mg}$ cortisol. Dexamethasone is believed to be 30 times more potent, by anti-inflammatory effectiveness, than cortisol. Thus, by dose-sensitive feedback potency, the two corticosteroids differed less than might have been predicted.

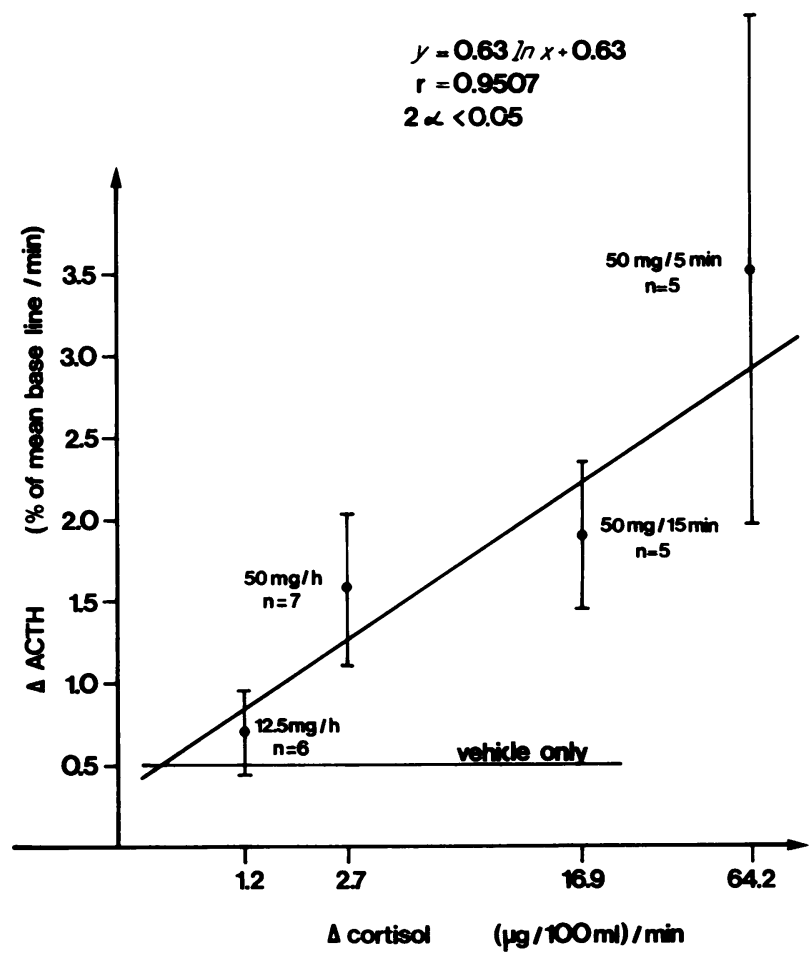

FIGURE 5 Relationship between the logarithm of the rate in increase in cortisol concentrations per minute (cortisol concentration at the end of the infusion period minus starting levels divided by the interval) and the corresponding decrease of ACTH levels per minute (ACTH starting level minus ACTH level at the end of the infusion period divided by the interval). When the infusion period exceeded $15 \mathrm{~min}$, only the interval between 0 and 15 min was considered. The minimal effective rate of increase in cortisol concentration was estimated to be $\cong 0.6(\mu \mathrm{g} / 100 \mathrm{ml}) / \mathrm{min}$ by plotting the data observed with vehicle only on the regression line. 


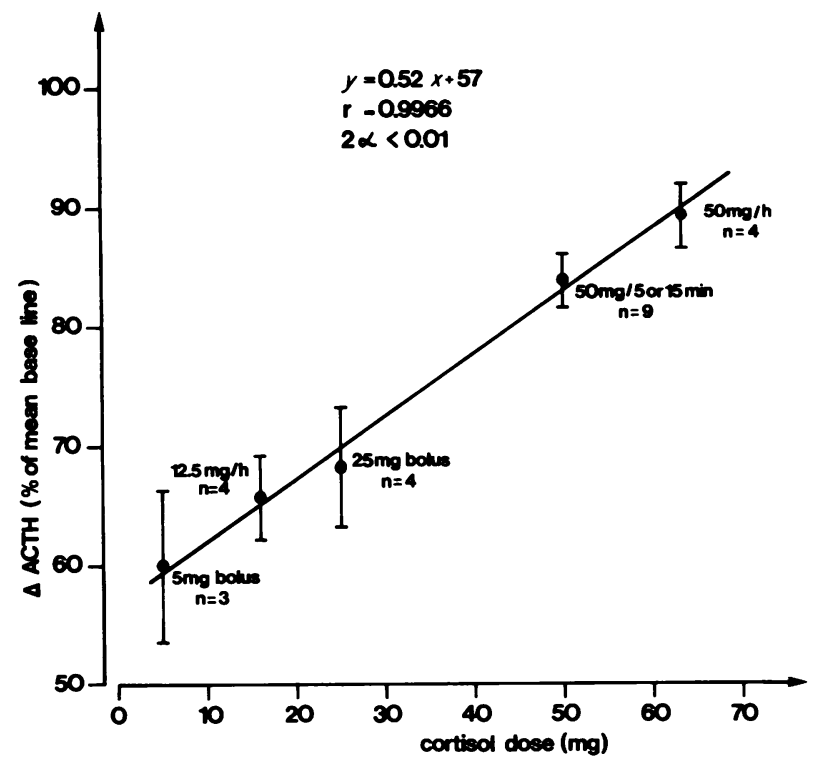

FIGURE 6 Relationship between the decrease in ACTH values $75 \mathrm{~min}$ after cortisol administration (ACTH starting levels minus ACTH levels at the 75th min) and the cortisol dose administered during this time, irrespective of the protocol.

\section{DISCUSSION}

Our data, taken together, indicate the presence of two separate processes for inhibition on nonstress ACTH hypersecretion by corticosteroids in patients with Addison's disease. A first decline in ACTH levels occurred without delay whenever, and as long as, cortisol concentrations were rising. The degree of inhibition of ACTH levels correlated with the rate of increase in cortisol concentrations. This mechanism may be designated differential or rate-sensitive glucocorticoid feedback mechanism. The results with bolus injections did not show the expected rate-sensitivity. We believe that a bolus may not produce a strong rate signal because it can be too brief. If the half-life of plasma ACTH is considered to be some minutes, even the strongest signals will produce no measurable changes in ACTH levels when the duration of the signal is only some seconds. A second mechanism became manifest with a delay time of $\cong 30 \mathrm{~min}$; in this case, the degree of inhibition was proportional to the dose of cortisol, however administered. This mechanism may therefore be designated integral or dose-sensitive feedback mechanism. Others have reported that the duration of inhibition is proportional to the dose as well $(4,6)$. This assumption, however, has not been tested by us.

The concept that the inhibitory effects of corticosteroids act by two feedback mechanisms, which we have found in man, is in good agreement with the results of experiments in which the inhibition of stressinduced ACTH secretion by glucocorticoids has been

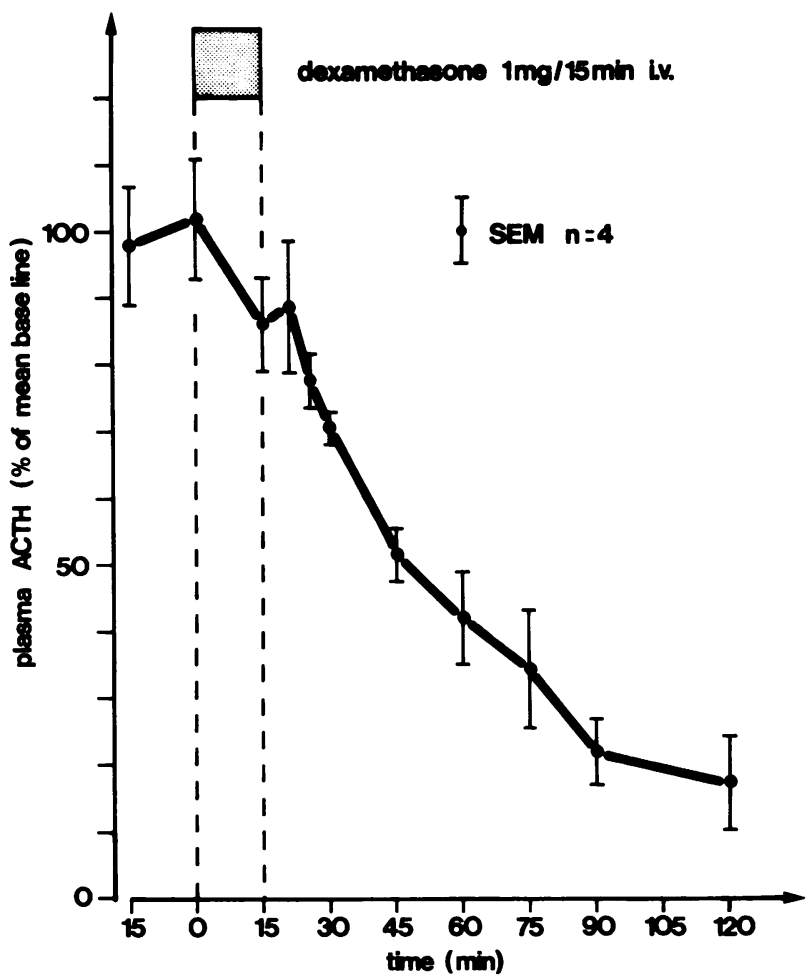

FIGURE 7 Time course of ACTH levels in response to infusion of $1 \mathrm{mg}$ dexamethasone during $15 \mathrm{~min}$ in patients with Addison's disease.

studied in the rat (3-9). In these experiments, there was a silent period of at least $1 \mathrm{~h}$ between the two phases of inhibition. The absence of a silent period in our experiments may be because nonstress and stress-induced ACTH secretion are dissociated on the basis of sensitivity to negative corticosteroid feedback effects (10-12). In more recent experiments, corticosteroid feedback mechanisms were investigated at the hypothalamic level with the technique of the rat hypothalamus in vitro $(18,19)$; both fast and delayed feedback effects on secretion of corticotropin-releasing factor were demonstrated with doses of corticosteroids within, or near, the physiologic range. The results suggested that the fast feedback mechanism acted via the inhibition of release, whereas the delayed feedback mechanism acted via inhibition of both synthesis and release. Sato et al. (20) reached a similar conclusion based on in vivo studies. These findings provide a possible explanation for the paradoxical overshoot in ACTH levels immediately after cessation of the corticosteroid infusion in our experiments. During the period of rapid feedback, corticotropin-releasing factor could have accumulated in the hypothalamus. When the rapid feedback mechanism stopped and the delayed feedback mechanism was not yet effective, more corticotropin-releasing factor was available to induce an increase in ACTH levels. 
The anti-inflammatory potency of dexamethasone is 30 times greater than that of cortisol in human beings (13). In our experience, a dose of dexamethasone equipotent to $30 \mathrm{mg}$ cortisol had no significant rate-sensitive feedback effects on ACTH secretion, and the dosesensitive feedback effects were less than expected from the relative potency. The great potency of dexamethasone as an inhibitor of ACTH release may be revealed only by studying the duration of inhibition. Yates and Maran (21) found that dexamethasone failed completely to inhibit ACTH release when it was given to rats from $15 \mathrm{~s}$ to a few minutes before a stressor. Dexamethasone-21-phosphate, used by these authors and by us, is more water soluble than is corticosterone, so it may not enter cells as rapidly as does the natural corticosteroid, which dissolves readily in lipid components of cell membranes. In these experiments, dexamethasone had to be given $1-2 \mathrm{~h}$ before a stressor to reveal its great inhibitory potency. On the other hand, Jones and Hillhouse (19) classified dexamethasone as an agonist of fast feedback with the hypothalamus in vitro.

Recently, we could demonstrate that in patients who have been totally adrenalectomized for treatment of Cushing's disease (hypercortisolism secondary to inappropriate ACTH secretion by the pituitary) infusion of cortisol induced a paradoxical rise in plasma ACTH levels of $\cong 400 \%$ (22). This rise occurred only during the first $15 \mathrm{~min}$ of an infusion of $50 \mathrm{mg} / \mathrm{h}$ cortisol. Later, ACTH levels decreased, but did not fall far below starting levels. A more detailed examination of feedback effects of cortisol on ACTH secretion with similar protocols as in this study may provide a better understanding of the disturbance of the corticosteroid feedback mechanism characteristic for Cushing's disease.

Finally, we want to point out that rate sensitivity is a common property of many biological systems. Unidirectional rate sensitivity was first discovered in a number of biological reflex systems as described by Clynes (23). There are striking similarities to the derivative and static mechanisms for insulin infusion, which are necessary to normalize glucose concentrations in diabetics by artificial beta cell $(24,25)$.

\section{ACKNOWLEDGMENTS}

We are deeply indebted to Professor F. E. Yates for his comments, criticism, and encouragement. The expert technical assistance of Mrs. Ulla Winkler is gratefully acknowledged.

This study was supported by Deutsche Forschungsgemeinschaft, Bonn, SFB 87, West Germany.

\section{REFERENCES}

1. Sayers, G., and M. Sayers. 1947. Regulation of pituitary adrenocorticotrophic activity during the response of the rat to acute stress. Endocrinology. 40: 265-273.
2. Yates, F. E., S. E. Leeman, D. W. Glenister, and M. F. Dallman. 1961. Interaction between plasma corticosterone concentration and adrenocorticotropin-releasing stimuli in the rat: evidence for the reset of an endocrine feedback control. Endocrinology. 69: 67-80.

3. Dallman, M. F., and F. E. Yates. 1969. Dynamic asymmetries in the corticosteroid feedback path and distribution-metabolism-binding elements of the adrenocortical system. Ann. N. Y. Acad. Sci. 156: 696-721.

4. Takebe, K., H. Kunita, M. Sakakura, Y. Horiuchi, and K. Mashimo. 1971. Suppressive effect of dexamethasone on the rise of CRF activity in the median eminence induced by stress. Endocrinology. 89: 1014-1019.

5. Jones, M. T., F. R. Brush, and R. L. B. Neame. 1972. Characteristics of fast feedback control of corticotrophin release by corticosteroids. J. Endocrinol. 55: 489-497.

6. Jones, M. T., E. M. Tiptaft, F. R. Brush, D. A. N. Fergusson, and R. L. B. Neame. 1974. Evidence for dual corticosteroid-receptor mechanism in the feedback control of adrenocorticotrophin secretion. J. Endocrinol. 60: 223233.

7. Bucher, B., B. Koch, and C. Mialhe. 1973. Sur l'existence d'un mécanisme de "feedback rapide" ACTH-corticostérone. J. Physiol. (Paris). 66: 199-209.

8. Sakakura, M., Y. Saito, K. Takebe, and K. Ishii. 1976. Studies on fast feedback mechanism by endogenous glucocorticoids. Endocrinology. 98: 954-957.

9. Engelland, W. C., J. Shinsako, C. M. Wingel, J. VernikosDanellis, and M. F. Dallman. 1977. Circadian pattern of stress-induced ACTH secretion are modified by corticosterone response. Endocrinology. 100: 138-147.

10. Zimmermann, E., and V. Critchlow. 1969. Suppression of pituitary-adrenal function with physiological plasma levels of corticosterone. Neuroendocrinology. 5: 183-192.

11. Zimmerman, E., and V. Critchlow. 1969. Negative feedback and pituitary-adrenal function in female rats. Am. J. Physiol. 216: 148-155.

12. Ács, T., and E. Stark. 1975. Effect of cortexolone on the feedback action of dexamethasone. Experientia (Basel). 31: 1365-1366.

13. Eisenstein, A. B. 1967. The Adrenal Cortex. Little, Brown \& Company, Boston. 1st edition. 370.

14. Fehm, H. L., K. H. Voigt, and E. F. Pfeiffer. 1972. Problems and artefacts in ACTH-assay. Horm. Metab. Res. 4: 477-481.

15. Voigt, K. H., H. L. Fehm, R. Reck, and E. F. Pfeiffer. 1974. Spontaneous and stimulated secretion of QUSOextractable immunoassayable ACTH in man. Klin. Wochenschr. 52: 516-521.

16. Murphy, B. E. P. 1976. Some studies of the proteinbinding of steroids and their application to the routine micro and ultramicro measurement of various steroids in body fluids by competitive protein-binding radioassay. $J$. Clin. Endocrinol. Metab. 27: 973-990.

17. Snedecor, G. W., and W. G. Cochran. 1973. Statistical Methods. The Iowa State University Press, Ames, Iowa. 6th edition. 135- 195.

18. Hillhouse, E. W., and M. T. Jones. 1976. Effect of bilateral adrenalectomy and corticosteroid therapy on the secretion of corticotrophin-releasing factor activity from the hypothalamus of the rat in vitro. J. Endocrinol. 71: $21-30$.

19. Jones, M. T., and E. W. Hillhouse. 1976. Structureactivity relationship and the mode of action of corticosteroid feedback on the secretion of corticotrophin-releasing factor (corticoliberin). J. Steroid Biochem. 7: 1189-1202.

20. Sato, R., M. Sato, J. Shinsako, and M. F. Dallman. 1975. 
Corticosterone-induced changes in hypothalamic corticotrophin-releasing factor (CRF) content after stress. Endocrinology. 97: 265-274.

21. Yates, F. E., and J. W. Maran. 1974. Stimulation and inhibition of adrenocorticotrophin release. Handb. Physiol. 4 (Section 7, Endocrinology): 367-404.

22. Fehm, H. L., K. H. Voigt, R. E. Lang, K. E. Beinert, G. W. Kummer, and E. F. Pfeiffer. 1977. Paradoxical ACTH response to glucocorticoids in Cushing's disease. N. Engl. J. Med. 297: 904-907.

23. Clynes, M. 1961. Unidirectional rate sensitivity: A bio- cybernetic law of reflex and humoral systems as physiologic channels of control and communication. Ann. N.Y. Acad. Sci. 92: 946-968.

24. Kerner, W., C. Thum, G. Tamás jun., W. Beischer, A. H. Clemens, and E. F. Pfeiffer. 1976. Attempts at perfect normalization of glucose tolerance of severe diabetics by artificial beta cell. Horm. Metab. Res. 8: 256-261.

25. Clemens, A. H., P. H. Chang, and R. W. Myers. 1977. The development of biostator, a glucose controlled insulin infusion system (GCIIS). Horm. Metab. Res. 7(Suppl.): 23-33. 